\title{
Evaluación de la inteligencia emocional en cuidadoras de guardería infantil
}

\author{
Evaluation of emotional intelligence in daycare caregivers
}

\author{
Oscar Octavio Acosta-López, , Jorge G. Escobar-Torres ${ }^{b}$, Dayana Luna-Reyes ${ }^{c}$, Santos Noé \\ Herrera-Mijangos ${ }^{d}$, Gerardo Hurtado-Arriaga ${ }^{e}$, Flor de María Gil-Bernal ${ }^{f}$
}

\begin{abstract}
:
The emotional intelligence is an important topic that offers huge advantages and skills in the life of people, however, rarely is considered and used in institutions, especially in kindergarten where the children are in developing and are similar to sponges that are absorbing everything. For that reason, this investigation make an evaluation of the emotional intelligence in 12 caregivers of kindergarten belonging to The Instituto Mexicano del Seguro Social (IMSS) through the Mayer-Salovey-Caruso Emotional Intelligence Test (MSCEIT) to know their levels and to adapt an intervention to adjustment to their needs. That intervention works in areas of the emotional intelligence (emotional perception, emotional facilitation, emotional understanding and emotional management), self-esteem, social skills and assertiveness. The results show a total of 8 caregivers (67\%) in the total rating (CIE) got scores lower than 90 points in the MSCEIT scale, what corresponds to the level of emotional intelligence called like "aspect to develop" which means that more than the half of the caregivers need improve in the area of emotional intelligence.
\end{abstract}

Keywords:

Emotional intelligence, education, nursery, caregivers.

Resumen:

La inteligencia emocional es un tema de gran importancia que ofrece grandes ventajas y habilidades en la vida de las personas, sin embargo, muy pocas veces es considerado y empleado en las instituciones, especialmente en las guarderías donde las niñas y niños están en desarrollo y son semejantes a esponjas que se encuentran absorbiendo todo. Por tal motivo, esta investigación hace una evaluación de la inteligencia emocional a 12 cuidadoras de una guardería perteneciente al Instituto Mexicano del Seguro Social por medio del Test de Inteligencia Emocional Mayer-Salovey-Caruso (MSCEIT) para conocer sus niveles y adaptar una intervención que se ajuste a sus necesidades. Dicha intervención trabaja las áreas de la inteligencia emocional (percepción, facilitación, comprensión y manejo), autoestima, habilidades sociales y asertividad. Los resultados muestran que un total de 8 cuidadoras (67\%) en su calificación total (CIE) obtuvieron puntuaciones menores a 90 en la escala del MSCEIT, lo que corresponde a nivel de inteligencia emocional denominado como "Aspecto a desarrollar", lo que significa que más de la mitad de las cuidadoras de guardería necesitan mejorar en el área de inteligencia emocional.

Palabras Clave:

Inteligencia emocional, educación, guardería, cuidadoras.

\section{Introducción}

\footnotetext{
a Universidad Autónoma del Estado de Hidalgo, https://orcid.org/0000-0002-4440-6104 , Email: blackened_dyers@hotmail.com

b Autor de Correspondencia, Universidad Autónoma del Estado de Hidalgo, http://orcid.org/0000-0002-9598-8781, Email: jorge_escobar3257@uaeh.edu.mx

c Universidad Autónoma del Estado de Hidalgo, https://orcid.org/0000-0001-9687-2521, Email: dayis2902@ gmail.com

d Universidad Autónoma del Estado de Hidalgo, https://orcid.org/0000-0001-6567-0986, Email: psicologonoe@yahoo.com.mx

e Universidad Autónoma del Estado de Hidalgo, https://orcid.org/0000-0003-4442-836X, Email: ghurtado@uaeh.edu.mx

${ }^{\text {f }}$ Universidad Autónoma del Estado de Hidalgo, http://orcid.org/0000-0001-7756-3125, Email: florerari@yahoo.com

Fecha de recepción: 26/10/2020, Fecha de aceptación: 26/01/2021, Fecha de publicación: 05/06/2021 
El Fondo de las Naciones Unidas para la Infancia $(\text { UNICEF })^{1}$ en su informe de 2015, destaca que de los 0 a los 5 años de edad del infante es la etapa fundamental para el desarrollo de capacidades físicas, intelectuales y emotivas. Durante esta los niños desarrollan en gran medida su seguridad y autoestima motivo por el cual las condiciones en las que se encuentren inmersos influirán en su crecimiento.

Los bebés comienzan su integración a las diversas áreas de la vida en sociedad a través de las instituciones materno-educativas que existen en gran parte del mundo. Dichos centros surgen debido a las nuevas demandas que hay en la vida laboral y familiar, de tal modo que se ven reflejadas en necesidades respecto a la forma de crianza. En los centros educativos generalmente la educación es colectiva, por ello, la crianza de los padres se ve compartida con otras personas que influyen en la vida de sus hijos. Para las niñas y niños que acuden a centros infantiles a edades tempranas se presentan nuevos retos como los planteados por la Secretaria de Educción Pública en 2017, siendo estos la separación temprana de sus padres por varias horas al día, el hecho de que la cuidadora o cuidador están a cargo de un mayor número de niñas y niños. De igual modo contempla las nuevas relaciones de comunicación y convivencia con la familia, agentes educativos e iguales. ${ }^{2}$

Por lo anterior, el rol de las cuidadoras de guardería es fundamental debido a que en ellas recae una gran responsabilidad que es el cuidado de las y los más pequeños, que se encuentran necesitados de atención, cariño y comprensión ante la temprana y dura separación de sus padres en periodos prolongados. Es por ello que se necesita de un ambiente adecuado y favorable donde se cuente con los espacios correspondientes para el correcto desarrollo, además de personal en constate capacitación que cumpla con el perfil.

La piedra angular de esta investigación es evaluar la inteligencia emocional de las personas encargadas del cuidado de los niños y la forma en que esta inteligencia se ve influenciada por la diversidad de vida de las trabajadoras de una Guardería perteneciente al Instituto Mexicano del Seguro Social (IMSS). Asimismo, comparar la forma en cómo los distintos niveles de inteligencia se ven influidos por distintos factores como son la edad, estado civil y escolaridad.

\section{Marco Teórico}

El concepto de inteligencia ha recorrido un largo camino, desde su concepción hasta la adaptación a la visión multidimensional de inteligencia humana, Bello et al. en 2010 plantea que ésta dirige su mirada a factores personales y contextuales donde recae la importancia de la actuación en la vida cotidiana. La propuesta de inteligencia emocional nace como una solución a las demandas de las condiciones sociales, esta se ha convertido en una necesidad en varios escenarios ya que pretende la armonía entre la emoción y la cognición. Nuestras emociones son la guía cuando tenemos que enfrentar momentos o situaciones difíciles motivo por el cual están inmersas en todas nuestras actividades de la vida. De este modo, Goleman en 1999 concibe a la Inteligencia Emocional como la capacidad de controlar y regular los propios sentimientos y los de los demás, así como la habilidad de utilizarlos como una guía para el pensamiento y de la acción que se puede concebir como un predictor del éxito en la vida de las personas y que se encuentra al alcance de todos. ${ }^{3,4}$

Por otro lado, la investigación de Ardila en 2011 menciona que las distintas concepciones respecto a la inteligencia se ven reflejadas en relación con los problemas en su definición, a sus características, las formas de medición, entre otros; y la mayoría de las definiciones de la inteligencia se clasifican bajo distintas visiones filosóficas, pragmáticas, factoriales y operacionales. Así, autores como Ebbinghaus en 1885 consideraba a la inteligencia como la capacidad de adaptarse a nuevas situaciones, mientras que Binet en 1983 creía que la inteligencia era determinada por la memoria, percepción y atención. Por otra parte, Sternberg en 1990 concebía que la inteligencia se basaba en tres categorías: habilidades analíticas, creativas y prácticas. En este orden de ideas, Ardila ${ }^{5}$ plantea también que los seres humanos cuentan con habilidades de comportamiento complejos y que la inteligencia posibilita la adaptación al medio ambiente, por consiguiente, reflexiona sobre la inteligencia y la adaptación, ya que ambas están sumamente ligadas y son necesarias para dar respuesta a los acontecimientos de la vida. ${ }^{5,6,7,8}$

Gardner de la Universidad de Harvard, realizó entre 1979 y 1983 un estudio sobre la naturaleza del potencial humano y su realización, concluyendo con la publicación de la obra "Frames of Mind" donde propuso que los individuos tienen alrededor de 8 inteligencias múltiples, siendo éstas la inteligencia lingüística-verbal, lógico-matemática, visual-espacial, musical, corporal cinestésica, intrapersonal, interpersonal y naturalista. ${ }^{9}$

Por otra parte, Salovey et al. en 1990 definen a la inteligencia emocional como la habilidad para percibir las emociones, así como sentimientos propios y de los demás, asimismo de identificarlos y emplearlos como una guía de los pensamientos y las acciones. ${ }^{10}$

En síntesis, estas propuestas reflejan la evolución y las distintas concepciones que se han tenido en relación con la inteligencia, desde centrarse en la tradicional perspectiva de su medición hasta considerar la 
diversidad de inteligencias que pueden existir en las personas.

En cuanto a las emociones, el Diccionario de Psicología de Galimberti en 2002 define a la emoción como una reacción afectiva intensa de aparición penetrante y de corta duración que es repuesta de un estímulo ambiental.

En este sentido Goleman en 1999, plantea que las emociones se refieren a "un sentimiento y a los pensamientos, los estados biológicos, los estados psicológicos y el tipo de tendencias a la acción que lo caracterizan" (p242). Por ello Barchard et al. en 2004 menciona que las personas que han logrado desarrollar habilidades emocionales pueden comprender y expresar sus propias emociones, identificar las emociones de los demás, regular el afecto y, utilizar estados de ánimo y emociones para impulsar conductas adaptativas. ${ }^{11,4,12}$

Referente a las relaciones interpersonales, Mayer et al. en el 2000 plantean que las personas inteligentes emocionalmente, además de desenvolverse con sus propias emociones, también logran relacionarse con las emociones de las otras personas. Ello, tendrá relevancia para el establecimiento, mantenimiento y calidad de las relaciones interpersonales en el ámbito psicológico de los estudiantes ya que aquellos que presentan mayor inteligencia emocional, tienden a mostrar menos estrés y ansiedad, aunque se encuentren sometidos a actividades que se consideren de mayor presión. Por otra parte, en el área de rendimiento académico, la inteligencia emocional funge como moderador de habilidades cognitivas sobre el rendimiento que los estudiantes puedan presentar. Finalmente y en relación con conductas disruptivas, las personas con un mayor nivel de inteligencia emocional tienen mayor tendencia a no generar algún tipo de adicción o dependencia. ${ }^{13}$

Ahora bien, el término de cuidador va desde la concepción simple de ser la persona encargada de acompañar al individuo que "requiere" de una peculiar atención, hasta tomar decisiones sobre otra persona. Es importante destacar tal como lo hacen Rivas et al. en 2011 que la labor o labores son las que dan sentido al cuidador, entre estas se encuentran: cuidado, asistencia, tareas de apoyo físico y emocional, entre otras. Para los mismos autores, las tareas que destacan del cuidador se centran en tres ejes de acción: las responsabilidades del cuidado mismo, la participación en la toma de decisiones conjuntas a la persona cuidada y la de poner en marcha la solidaridad con el que se encuentra sufriendo. De igual forma, es importante considerar que cuando se considera el cuidador, también se debe de pensar en el contexto donde éste toma significado, como es lugar, tiempo, cultura y situación. ${ }^{14}$

En México, el Instituto Nacional de Estadística y Geografía (INEGI) [citado en Ramírez] en 2017 estima que por lo menos en una mitad de los hogares en México vive alguna persona que requiere cuidado (desde niños hasta adultos mayores). El $97 \%$ de los cuidadores son mujeres con sueldos de uno y dos salarios mínimos, aproximadamente 23.9 pesos por hora trabajada. Un $40 \%$ de las cuidadoras tienen entre 15 y 29 años de edad. Por otro lado, el INEGI en 2018, estimó que en México 3.5 millones de niños y niñas de 0 a 6 años son cuidados en una guardería, estancia infantil o por terceras personas, cifra equivalente al $25 \%$ de la población infantil en dicho rango de edad. El 16.3\% fueron atendidos en una guardería, mientras que el $83.7 \%$ estuvo al cuidado de una persona, destacando la abuela como la principal cuidadora en un $65.6 \%$ de los casos. ${ }^{15,16}$

Dentro de los manuales ofrecidos por el Instituto Mexicano del Seguro Social en 2019 se enlistan las actitudes del educador, que son la manera en que las y los cuidadores se deben relacionar con los niños, ya que esto es determinante para logar las metas educativas propuestas. En este sentido, la investigación realizada por García en 2012 resalta la importancia de que los educadores trabajen su inteligencia emocional, se necesita que se conozcan a sí mismos y que estén más en contacto con sus emociones para después comprender las de los demás. Por ende, se necesita considerar a la educación como un proceso integral donde los aprendizajes y emociones están íntimamente relacionados. ${ }^{17,18,19}$

Además la investigación de Cejudo et al. en 2017 destaca las características personales que pueden ser necesarias para el trabajo como docentes. La gestión emocional de los demás, las habilidades de relación y el control de la impulsividad se consideran como dimensiones de la inteligencia emocional de carácter interpersonal. Sin embargo, se encuentran características personales que pueden ser menos necesarias para el desempeño del trabajo de un docente como es el caso de la percepción emocional propia, la regulación emocional propia y la gestión del estrés que son clasificadas como dimensiones de carácter intrapersonal. Los resultados muestran un efecto colateral de que la mejora en la inteligencia emocional en los maestros podría tener beneficios en el proceso de enseñanza-aprendizaje. También se encontró relación significativa en que las maestras tienden a estar más preocupadas en aspectos relacionados con la motivación que sus compañeros varones y, de igual forma, no se encuentran diferencias significativas en función de la antigüedad. Estos resultados son importantes debido a la relación que se destaca y que se busca indagar en esta investigación. ${ }^{20}$

Otro punto a destacar es el de Cabello et al. en 2010, quienes plantean que se cuentan con escasos programas socioemocionales encargados de fomentar el desarrollo de la inteligencia emocional en los profesores. 
Es así como se contempla en los docentes que la práctica de la enseñanza se ve comprometida con las cargas emociones que puedan trasmitir hacia los estudiantes. ${ }^{21}$

En cuanto al aprendizaje, Díaz en 2012 lo define como "una serie de procesos biológicos y psicológicos que ocurren en la corteza cerebral que, gracias a la mediatización del pensamiento, llevan al sujeto a modificar su actitud, habilidad, conocimiento e información, así como sus formas de ejecución, por las experiencias que adquiere en la interacción con el ambiente externo, en busca de dar respuestas adecuadas" (p6), a su vez, menciona que la práctica juega un papel de suma importancia para la obtención de aprendizaje pues, ayudará a que el cambio producido en el proceso de enseñanza sea duradero. Bajo esta línea, la Secretaria de Educación Pública en 2004 menciona que tener conocimiento de las distintas maneras en que los alumnos aprenden permite buscar los recursos más adecuados que se amolden a dichos estilos facilitando de esa manera el aprendizaje, asimismo se debe considerar que aunque los estilos de aprendizaje son relativamente estables, también son susceptibles a cambios o mejoras, dependiendo de las distintas situaciones. Además Piaget en 1977 afirma que el aprendizaje conlleva un proceso de asimilación de los conocimientos obtenidos. El individuo adapta y reorganiza la información creando nuevas estructuras mentales a través estímulos y de la interacción con el medio. El individuo obtiene conocimiento por medio de las experiencias concretas en el entorno en que se desarrolla, relacionando la información nueva con información adquirida con anterioridad. ${ }^{22,23,24}$

Por otra parte Casassus en 2007 menciona que el rol del docente implica una carga de trabajo emocional y cognitiva, debido a que se exige sensibilidad de las emociones propias y ajenas, es importante señalar que el manejo adecuado de éstas permite mejorar la calidad de relaciones interpersonales. Por ende, existen mayores posibilidades que los docentes con mayor nivel de inteligencia emocional muestren interés a poseer características personales adecuadas para garantizar un proceso de enseñanza-aprendizaje de calidad. Asimismo, es importante considerar que una parte significativa de las deficiencias existentes en la educación y el área de las emociones está fuertemente ligado al tipo de sistemas que se manejen en el aula pues, se encuentran sujetos a límites establecidos que están obligados a cumplir; también es importante tener en cuenta que las necesidades de los profesores están estrechamente relacionadas a las necesidades de los alumnos. ${ }^{25}$

Finalmente Wong et al. en 2018 señalan que la expectativa hacia los maestros como el principal contribuidor para la educación de los niños puede impedir la participación de los padres en la educación de sus hijos. Los maestros deberían de mejorar la comunicación que se tiene con los padres, hablar de forma usual con éstos sobre el desempeño de los niños en el aula, con el objetivo de maximizar el potencial académico y de beneficiar su educación. ${ }^{26}$

\section{Objetivos}

General: Determinar las diferencias que hay entre cuidadoras de guardería respecto a su nivel de inteligencia emocional, la antigüedad en el puesto y sus características sociodemográficas.

Específicos:

1.-Medir el nivel de inteligencia emocional de las cuidadoras de guardería.

2.-Comparar la inteligencia emocional de las cuidadoras de guardería respecto a sus diferencias sociodemográficas.

\section{Metodología}

Es un tipo de estudio no experimental, transversal exploratorio y descriptivo pues se indagaron las características de la Inteligencia Emocional en relación con la antigüedad, la edad y estado civil de las cuidadoras de guardería.

\section{Hipótesis}

\section{a. Conceptual}

De acuerdo con Luna et al. en 2016, el nivel de inteligencia emocional de las cuidadoras de guardería tiende a ser mayor en aquellas que presentan mayor grado de estudios, lo que también representa una desigualdad social en las oportunidades sociodemográficas de vida de las personas. ${ }^{27}$

Se presenta mayor nivel de inteligencia emocional obtenido a través del Test de Inteligencia Emocional Salovey-Mayer-Caruso en las cuidadoras que se encuentran en algún tipo de relación sentimental que las que se encuentran solteras.

Se presenta mayor nivel de inteligencia emocional obtenido a través del Test de Inteligencia Emocional Salovey-Mayer-Caruso en las cuidadoras que más jóvenes en comparación con aquellas que son mayores de 30 años.

Se presenta mayor nivel de inteligencia emocional obtenido a través del Test de Inteligencia Emocional Salovey-Mayer-Caruso en las cuidadoras que tienen mayores niveles de estudios en comparación a aquellas que no presentan más grados de escolaridad.

Se presenta mayor nivel de inteligencia emocional obtenido a través del Test de Inteligencia Emocional Salovey-Mayer-Caruso en las cuidadoras que 
tienen menor nivel de antigüedad en el puesto en comparación con las cuidadoras que llevan más de 6 años laborando en la guardería.

\section{b. Estadística}

Alterna $(\mathrm{H} 1)$ : $\mathrm{Si}$ existen diferencias estadísticamente significativas entre el nivel de inteligencia emocional de las cuidadoras de guardería respecto a sus características sociodemográficas. Nula $(\mathrm{H} 0)$ : No existen diferencias estadísticamente significativas entre el nivel de inteligencia emocional de las cuidadoras de guardería respecto a sus características sociodemográficas.

\section{Instrumento para la recolección de la información}

El Test de inteligencia emocional Mayer-Salovey-Caruso MSCEIT de 2009, evalúa la inteligencia emocional dividida en dos áreas: experiencial y estratégica. Además, proporciona puntuaciones en las cuatro áreas principales de la inteligencia emocional que son: percibir emociones con precisión, utilizar emociones para facilitar el pensamiento, resolución de problemas y creatividad, comprender emociones y, manejar emociones para el crecimiento personal. La prueba está compuesta por 141 Ítems que arrojan una puntuación total CIE además de puntuaciones en las dos áreas evaluadas: inteligencia emocional experiencial (CIEX) e inteligencia emocional estratégica (CIES), asimismo brinda puntuaciones de cada una de las cuatro ramas evaluadas en estas áreas (Percepción emocional, Facilitación emocional, Comprensión emocional y Manejo emocional). La versión de investigación de MSCEIT presenta un índice de fiabilidad dos mitades satisfactoria, de 0.93 en la puntuación total y de $0.92,0.87,0.79,0.87$ en sus ramas respectivamente. ${ }^{28}$

Extremera et al. en 2006 al realizar la traducción y traducción inversa de la prueba concluyen que el MSCEIT v.2.0 ha demostrado tener buena confiabilidad y es apto para ser empleado en hispanohablantes, asimismo esta prueba permite la exploración de las diferencias culturales de la inteligencia emocional, por lo que es una prueba que puede ser empleada para futuras investigaciones. ${ }^{29}$

\section{Muestra}

Es un muestreo de tipo no probabilístico por conveniencia ya que donde se realizó la investigación se usó una muestra representativa de 12 cuidadoras de guardería que van de los 23 a los 50 años de edad y que llevan desde menos de 6 meses de antigüedad hasta más de 15 años de trabajo en la institución.

\section{Procedimiento}

En primera instancia, se solicitaron los permisos necesarios para el ingreso la Guardería perteneciente al Instituto Mexicano del Seguro Social (IMSS). Se tuvo una pequeña entrevista con la encargada de lugar donde se explicaron los objetivos de la investigación y también se trataron algunas inquietudes respecto a las cuidadoras. En la fecha acordada para la aplicación de la prueba, se llegó 15 minutos antes, siendo dos los aplicadores de la prueba; El tamaño de la institución no es muy grande, por ello los salones de la guardería son pequeños. El personal masculino es escaso, son dos hombres y se encuentran en el área de cocina mientras que el personal femenino es de aproximadamente 30 mujeres, 20 son cuidadoras dividas en turnos matutino y vespertino; 4 educadoras y 6 mujeres más que ocupan puestos en dirección, seguridad y enfermería. En el segundo piso se encuentra el espacio que nos brindaron para la aplicación de la prueba.

El salón de la aplicación era pequeño, las mesas y sillas no fueron las más apropiadas para el personal. Se contaba con 6 mesas por lo que se planeó iniciar con ese mismo número de trabajadoras y conforme fueran finalizando irían pasando las otras cuidadoras. Se les dio a conocer a las participantes el consentimiento informado, documento que señalaba aspectos de confidencialidad y uso de la información. De igual modo, se les hizo saber que no existía límite de tiempo para responder la prueba y que, si tenían alguna duda, con gusto se les apoyaría.

Muchas de las cuidadoras tomaron una postura bastante defensiva, hubo trabajadoras que se tomaron tiempo de más para contestar la prueba y sus compañeras expresaban su queja respecto a esta situación ya que argumentaban que hacían eso con el fin de estar menos tiempo a cargo de las niñas y niños. De igual forma, hubo trabajadoras que no quisieron responder la prueba debido a que estaba por concluir su horario laboral o no se contaba con personal que cubriera su puesto. También había trabajadoras que se percibían bastante presionadas por responder la prueba ya que tenían que checar la entrada laboral, por lo que debían presentarse a tiempo, para estar al tanto de sus grupos.

Cuando las participantes terminaban de contestar el test, se les agradecía su contribución y se tomaban los comentarios finales; de igual manera se les recordaba los números de contacto para cualquier duda 0 aclaración.

Posteriormente se procedió a dar cierre a la aplicación y a acomodar el espacio que nos fue brindado. Se acudió al área administrativa a dar gracias por el apoyo, a dar los últimos comentarios respecto a la prueba y se acordó brindar los resultados cuando estuvieran disponibles.

Las pruebas fueron analizadas y se obtuvieron las calificaciones de las cuidadoras. Posteriormente los 
resultados de las pruebas fueron introducidos en el programa de Excel para luego ser analizados en el Statistical Package for the Social Sciences (SPSS).

\section{Resultados}

El propósito de esta investigación fue identificar el nivel de inteligencia emocional de las cuidadoras de guardería y compararlo con sus características sociodemográficas.

\section{Características sociodemográficas de la muestra}

Se conformó por un total de 12 trabajadoras de una Guardería del Instituto Mexicano del Seguro Social, estas cuidadoras cumplen criterios de inclusión, principalmente que está en constante relación con las niñas y niños. Las variables sociodemográficas que se consideraron en el estudio fueron la edad, escolaridad, estado civil y antigüedad.

\section{Edad}

Como se muestra en la Figura 1, el rango de edad fue de los 23, que corresponde a la integrante más joven, a los 52 años que es la cuidadora de mayor edad. La media de edad fue de 34 y la mediana de 32.5. En la Figura 2, aproximadamente el $83.3 \%$ de las cuidadoras se encuentran en el rango de 20 a 40 años, lo que indica que gran parte de la población es relativamente joven. Mientras que el otro $16.7 \%$ superan los 40 años.

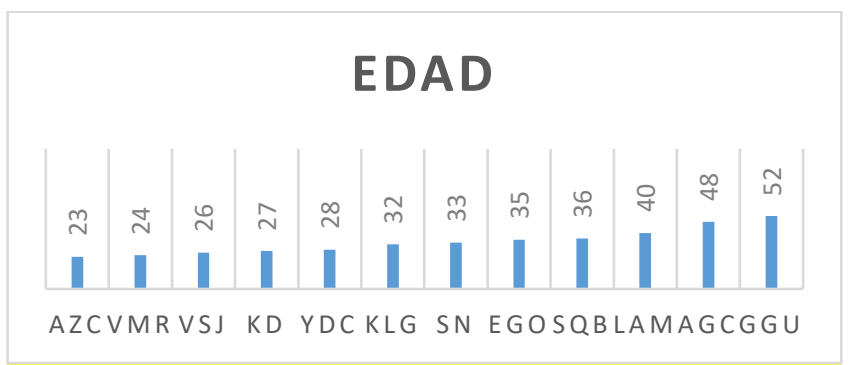

Figura 1. Edad de las cuidadoras de guardería

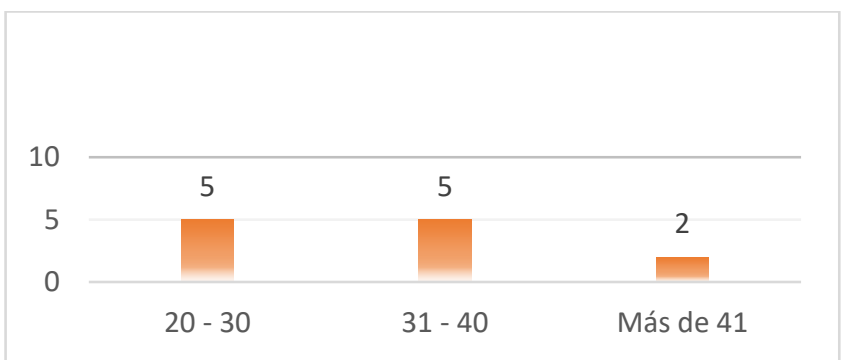

Figura 2. Rango de edad de las cuidadoras de guardería

Escolaridad
De la muestra se obtuvo que 4 cuidadoras (33.3\%) cuentan con estudio superiores, mientras que 3 (25\%) trabajadoras tienen nivel bachillerato y otras 3 (25\%) con secundaria. Por otra parte, 2 (16.7\%) cuidadoras tienen otro nivel de estudios del cual no especificaron como se muestra en la Figura 3. Por lo que se puede considerar que una buena parte de las cuidadoras no cuenta con la preparación necesaria para la adecuada función en la que están a cargo.

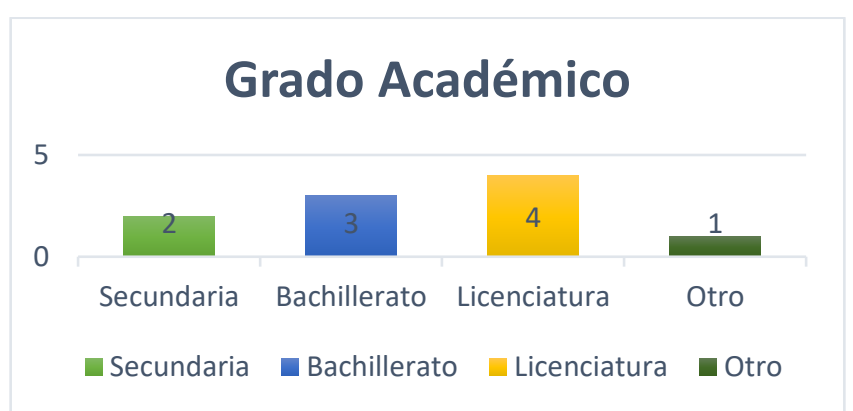

Figura 3. Grado académico de las cuidadoras de guardería.

Inferenciales (pruebas estadísticas)

Las calificaciones del MSCEIT ${ }^{28}$ se dividen en 5 rangos:

-0 a 70: Necesita mejorar

-70 a 90: Aspecto a desarrollar

-90 a 110: Competente

-110 a 130: Muy competente

-Más de 130: Experto

De acuerdo con las calificaciones obtenidas por el MSCEIT, el cual se aplicó a las 12 cuidadoras de guardería, se obtuvo lo siguiente: los puntajes máximos de CIE (Puntuación total de inteligencia emocional) corresponde a 98 mientras que la calificación más baja fue de 77 como se puede observar en la Figura 4. El promedio fue de 88.08; la mediana de 87.5 y una desviación estándar de 6.24.

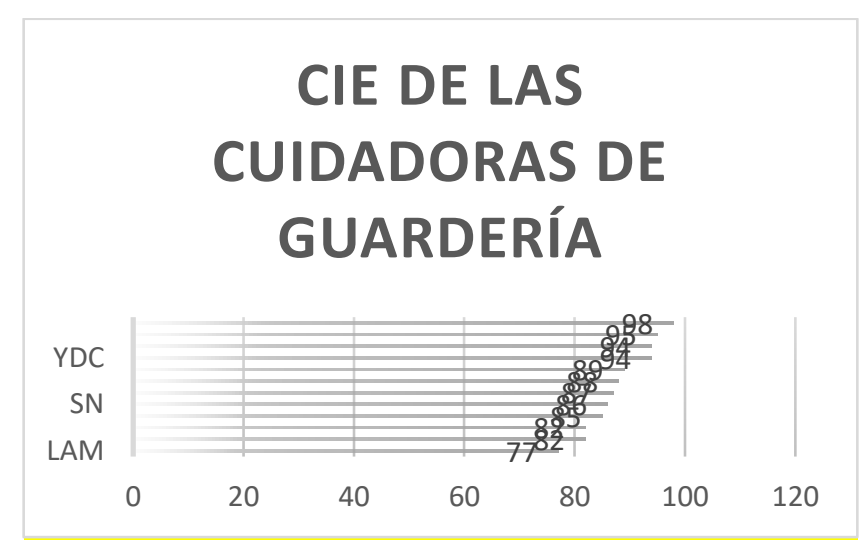


Figura 4. Puntuaciones totales (CIE).

Como se muestra en la Figura 5, un total de 8 cuidadoras $(67 \%)$ en su calificación total de CIE obtuvieron calificaciones menores a 90 puntos, lo que corresponde a nivel de inteligencia emocional denominado como "Aspecto a desarrollar", lo que significa que más de la mitad de las cuidadoras de guardería necesitan mejorar en el área de la inteligencia emocional. Por otra parte, 4 cuidadoras (33\%) obtuvieron calificaciones arriba de 90 puntos lo que significa que su inteligencia emocional es considerada como "competente" sin embargo, presentan áreas de oportunidad a desarrollar.

\section{Puntuación total (CIE)}

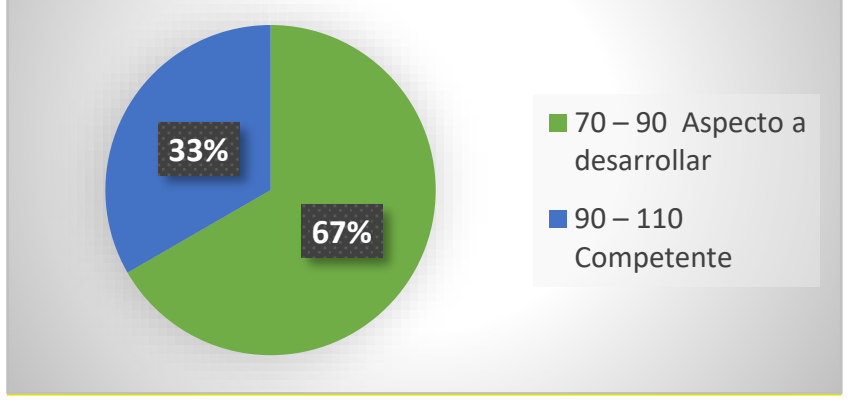

Figura 5. Puntuaciones totales (CIE).

\section{Resultados}

La inteligencia emocional destaca por las grandes ventajas que ofrece en múltiples aspectos de la vida a las personas, tales como educación, crianza, trabajo, relaciones interpersonales, etc. Sin embargo, en México es escasamente considerada en las áreas ya mencionadas, además de que son pocas las personas que la consideran o deciden practicarla y/o emplearla en sus vidas.

Por otra parte, el Instituto Mexicano del Seguro Social es una de las instituciones más trascendentes del país en lo que respecta al área de la salud y laboral. Es un organismo que integra programas para su mejora continua en sus diferentes servicios que ofrece, sin embargo, el ingreso laborar pasa por diversos filtros, perfiles de puesto además de cursos de capacitación con la finalidad de que cumplan las expectativas y objetivos de calidad propuestos.

Otro punto a destacar es que por medio de esta investigación se ha evidenciado que los requisitos de calidad no siempre se cumplen, debido a que en la recopilación de datos sociodemográficos se logró analizar que una parte significativa de las cuidadoras no cumplía con los estudios especializados para ejercer su cargo, y que los cursos de capacitación no cumplen los objetivos debido a la situación observada y analizada en la guardería. Estos incumplimientos además de influir en el clima laboral y el bienestar personal de las cuidadoras, se reflejan en las y los usuarios de la guardería que podrían recibir un mal cuidado; esto se debe considerar ya que los primeros años de vida de una persona son fundamentales para su desarrollo y también se debe considerar qué tanto las niñas y los niños se enfrentan a situaciones más complicadas ya que durante su infancia, la crianza y enseñanza se ve compartida con las cuidadoras quienes son las encargadas de la atención en una parte significativa del día.

Los resultados obtenidos por la prueba MSCEIT muestran que solo 4 cuidadoras se encuentran en el rango de "competentes" en el área de la inteligencia emocional, mientras que el resto de las cuidadoras (8 de ellas) se encuentran en el rubro de "aspecto a mejorar" lo que significa que las calificaciones obtenidas son bajas y resulta de suma importancia emprender acciones a favor del desarrollo y mejoramiento de la inteligencia emocional.

La autora Dolto en 1997 menciona que las niñas y niños que crecen en guardería obtienen ciertas habilidades como el contacto en grupo debido a su constante convivencia con otros usuarios, sin embargo, sus carencias se ven reflejadas con el contacto de sus padres ya que se ve interferida por agentes externos, además de la percepción que se tiene sobre el mundo externo. Por ello, la intervención de las cuidadoras es fundamental en la parte de la crianza y educación, ya que la proximidad existente hace que sean agentes importantes de la vida de las niñas y niños. ${ }^{30}$

Para finalizar, es importante mencionar que es fundamental establecer y aplicar una intervención cuyo objetivo fuera mejorar la inteligencia emocional de las cuidadoras de guardería; reflejándose así en el cuidado, crianza y educación de las y los usuarios del servicio. Asimismo se buscaría mejorar la relación que hay entre las trabajadoras y, al mismo tiempo, con los padres de familia. Para la intervención se requiere una validación por jueces, abordando diferentes temas por sesión de cada una de las áreas de la inteligencia emocional (percepción emocional, facilitación emocional, comprensión emocional y manejo emocional). Asimismo se complementa con otras áreas de oportunidad como son la autoestima, habilidades sociales y asertividad. Por tal motivo, se busca que la intervención también brinde herramientas y estrategias para el cuidado de las niñas y niños.

\section{Referencias}


[1] El Fondo de las Naciones Unidas para la Infancia. México UNICEF; 2015 [citado 9 de abril de 2019. Recuperado a partir de: https://www.unicef.org/mexico/spanish/

[2] Secretaría de Educación Pública. Aprendizajes Clave para la Educación Inicial. México: Secretaría de Educación Pública; 2017.

[3] Bello-Dávila Z, Rionda-Sánchez H, Rodríguez-Pérez M. La inteligencia emocional y su educación. Varona; 2010: 36-43.

[4] Goleman D. Inteligencia Emocional. Barcelona: Kairós; 1999.

[5] Ardila R. (2011). Inteligencia. ¿Qué sabemos y qué nos falta investigar? Ev. Acad. Colomb.Cienc. 2011; 35(134): 97-103.

[6] Ebbinghaus H. Memory a Contribution to Experimental Psychology. New York: Teachers College, Columbia University; 1885. Recuperado a partir de: https://archive.org/details/memorycontributi00ebbiuoft/page/n5

[7] Binet A. La inteligencia: su media y educación. Infancia y Aprendizaje: Journal for the Study of Education and Development. 1983; (22): 115-120.

[8] Sternberg R. Más allá del cociente intelectual: Una teoría triárquica de la inteligencia humana. España: Desclée de Brouwer; 1990.

[9] Gardner H. Estructuras de la Mente. México: Fondo de Cultura Económica; 1994.

[10] Salovey P, Mayer J. Emotional Intelligence. Imagination, Cognition and Personality; 1990; 9(3): 185-211

[11] Galimberti U. Diccionario de Psicología. México: Siglo Veintiuno; 2002.

[12] Barchard K, Hakstian A. The nature and measurement of Emotional Intelligence abilities: Basic dimensions and their relations with other cognitive ability and personality variables. Educational and Psychological Measurement. 2004; 64 (3): 437-462.

[13] Mayer J, Salovey P, Caruso D. Models of Emotional Intelligence. En: Salovey P, Brackett M, Mayer J. The Handbook of Intelligence New York: RJ Sternberg; 2000: 396-420.

[14] Rivas J, Ostinguín R. Cuidador: ¿concepto operativo o preludio teórico? Enfermería Universitaria. 2011; 8(1): 49-54. doi:http://dx.doi.org/10.22201/eneo.23958421e.2011.1.25471

[15] Ramírez M. Cuidadoras de personas dependientes en situación de precariedad [Internet]. La Izquierda Diario. Red Internacional en 7 Idiomas. 2017 [citado 2 de marzo de 2017]. Recuperado a partir de: https://www.laizquierdadiario.mx/Cuidadoras-de-personasdependiente s-en-situacion-de-precariedad

[16] Instituto Nacional de Estadística y Geografía. Sexta Edición de la Encuesta Nacional del Empleo y Seguridad Social (ENESS). Comunicado de Prensa; 2018: 1-21.

[17] Introducción a la Guía Técnica del Programa Educativo para el Servicio de Guarderías el Sistema IMSS [Internet]. Instituto Mexicano del Seguro del Social. [citado 15 de junio de 2019]. Recuperado a partir de: http://siag.imss.gob.mx/instalacionsiag/guarderias/guias/gt_economic a_completa.pdf

[18] Fases del Proceso de selección del Personal que Ingresa a Laboral al Instituto Mexicano del Seguro Social. SlideShare. 2015 [citado 15 de junio de 2019]. Recuperado a partir de:

https://es.slideshare.net/GatoImss/fases-del-proceso-de-ingreso-paratrabajar-en-el-imss

[19] García J. La Educación Emocional su Importancia en el Proceso de Aprendizaje. Revista Educación: 2012; 36 (1): 1-24.

[20] Cejudo J, López M. Importancia de la Inteligencia Emocional en la Práctica Docente: Un Estudio con Maestros. Psicología Educativa. 2017: 29-36.

[21] Cabello R, Ruiz D. Docentes emocionalmente inteligentes. Revista electrónica interuniversitaria de formación del profesorado. 2010; 13(1): 41-49.

[22] Díaz E. Estilos de Aprendizaje. Eídos. 2012; (5): 5-11.

[23] Secretaría de Educación Pública. Manual de estilo de aprendizaje. Ciudad de México: Dirección de Coordinación Académica; 2004.

[24] Piaget J. El Nacimiento de la Inteligencia del Niño. Madrid: Crítica; 1977.

[25] Casassus J. La educación del ser emocional. Chile: Cuarto Propio; 2007.

[26] Wong R, Ho F, Wong W, Tung K, Chow C, Rao N, Chan K, Ip P. Parental Involvement in Primary School Education: its Relationship with Children's Academic Performance and Psychosocial Competence through Engaging Children with School. Journal of Child and Family Studies, 2018: 1-12. doi:10.1007/s10826-017-10112

[27] Luna S, Rivera F, Ramos P. Ajuste psicológico y clasificación de mujeres cuidadoras de nietos y nietas y/o ascendientes en función de su inteligencia emocional. Apuntes de Psicología. 2016; 34 (2-3): 161-169.

[28] Mayer J, Salovey P, Caruso D. Test de Inteligencia Emocional Mayer-Salovey-Caruso. Madrid: Ediciones Tea; 2009.

[29] Extremera N, Fernández-Berrocal P, Salovey P. (2006). Spanish version of the Mayer-Salovey-Caruso Emotional Intelligence Test (MSCEIT). Version 2.0: Reliabilities, age and gender differences. Psicothema. 2006;18: 42-48.

[30] Dolto F. La Dificultad de Vivir Volumen II. Barcelona: Gedisa; 1997. 